科 学 通 报

\title{
条带织构装饰技术观察高分子液晶态 的向错形态
}

陈着俄 宋文辉 马于安** 金永泽 载人元

(中国科学院化学研究所, 北京 100080)

\section{美键词然带织构、向错形态、宫分子液晶态}

辛分子液晶态与小分子液晶态相似能表现出多种类型的中介相, 其中之一是向列相. 高 分子没向列根存在有向错结构, 它是由分子取向方向发生不连续变化而引起的. 在偏光显 微说下向列相星现二条和四条黑色条纹相交于一点所组成的纹影织构, 它们分别对应于强度 $s= \pm \frac{1}{2}$ 和 $s= \pm 1$ 两种类型的向错分子指向矢取向排列形式的一种光学效应. 近年来 人们经活高分子分子链弛潒时间较长和可以结晶的特性, 将高分子液晶态淬火冻结成液晶玻 璃态, 并应用片晶装饰技术在电子显微镜下直接观察高分子液晶态中各种类型的向 错形态. Thoinas $^{[1-3]}$ 等人首先应用片晶装饰技术在透射电镜下观察到热致聚芳酯冻结液晶 态中强度 $s= \pm \frac{1}{2}$ 类㤠的两种向错形态. $500 d^{[4]}$ 和董炎明[5]应用片晶装饰和化学刻蚀相结合技术在 扫推它镜下观察 $s= \pm \frac{1}{2}$ 和 $s=+1$ 的向错形态. 前文 ${ }^{[6]}$ 我们应用片晶装饰和 $\mathrm{RuO}_{4}$ 染色 相结合装术在透射电镜下洒察到热致聚茫酯液晶态中强度 $s= \pm \frac{1}{2}$ 和 $s= \pm 1$ 的全部两类 共六种问错形态. 本文发展了一种新的方法即条带织构装饰技术研究主链型热致高分子液 晶态中的向锴泡态, 在普通偏光显微镜下观察到了热致聚芳醌冻结液晶态沂存在的向错形态 及它们之间城互作用的状态。

\section{1 实 验部 分}

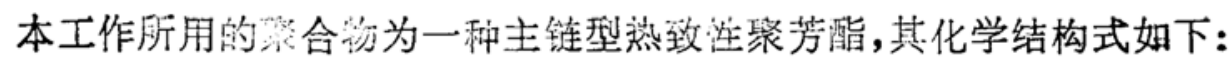<smiles>CC(F)(F)Oc1ccc(OC(O)(O)c2ccc(OC(=O)CCCCC(=O)Oc3ccc(C(=O)C(C)(C)C)cc3)cc2)cc1Cl</smiles>

以苯阳 - 四氯乙烷 $(1: 1)$ 混合液为溶剂在 $25^{\circ} \mathrm{C}$ 下测定其 $[\eta]=0.402 \mathrm{dL} / \mathrm{g} ;$ DSC 测定其液晶 态转代淂度为 $187^{\circ} \mathrm{C}$, 没有各向闰性化温度.

1992-07-29 收稿, 1992-10-19 收修改稿.

*中国科学蕰重大啡究资助项目.

** 北京航空航天大学 91 届毕业生. 
将聚合物粉末放在已恒温为 $200^{\circ} \mathrm{C}$ 的玻片上, 盖上一聚四氟乙烯薄膜, 加压集娍一厚度 为 $10 \mu \mathrm{m}$ 左右的薄膜试样, 然后将聚四㡘乙烯膜揭去使试样表面成自由面. 然后将试样放在 $260^{\circ} \mathrm{C}$ 温度下热处理 $10 \mathrm{~min}$ 后淬火至室温, 即得到具有条带织构的冻结液晶态试样. 试样置 于日产的 Olympus BH-2 型偏光显微镜下观察其向错形态.

\section{2 结果和讨论}

主链型热致性聚芳酯冻结液晶态在偏光显微镜下呈现出向列相液晶态的特征纹影织构, 它是液晶态中固有向错结构的一种光学效应. 在高分子液晶态中向错结构的形态可以用片晶 装饰技术使它呈现出, 在高分辨的电子显微镜下才能观察到它们的形态. 由于片晶尺寸很小 $(10 \mathrm{~nm})$, 在低分辨率的偏光显微镜下是无法看到的. 但幸运的是, 我们在研究主链型高分子 液晶态的热处理过程中发现 ${ }^{⿰ 刃 一}$, 热致性高分子液晶态随着热处理温度升高有些向错消失或合 并成新的大向错, 表现为向错点密度不断减小 (即向列微区不断增大). 当向列微区增大到一 定尺寸时将试样淬火冻结, 就可以得到呈现围绕向错点有规则排列成一定形状的条带织构, 这 些条带织构的尺寸为 $1 \mu \mathrm{m}$ 左右, 可以很容易在偏光显微镜观察到, 这些条带织构取向排列的 形式正好反欥了液晶态中各种向错结构的形态. 因此, 应用条带织构装饰技术表现主链型高 分子液晶态的句错结构形态不但可行, 门i且是一种简便有效的方法.

应用条带织构装饰技术首先必须确定条带织构与其分子链平均取向方向之间的对应关 系. 经研究指出 ${ }^{[8]}$ 在相邻条带中分子链取向排列分别偏离纤维轴方向成一定角度, 但是分子 链的平均取向方向是垂直于条带的长轴方向, 对主链型液晶高分子来说, 链的方向就是介晶单 元的指向矢方向. 条带织构与分子链平均取向之间的关系示于图 1 中.

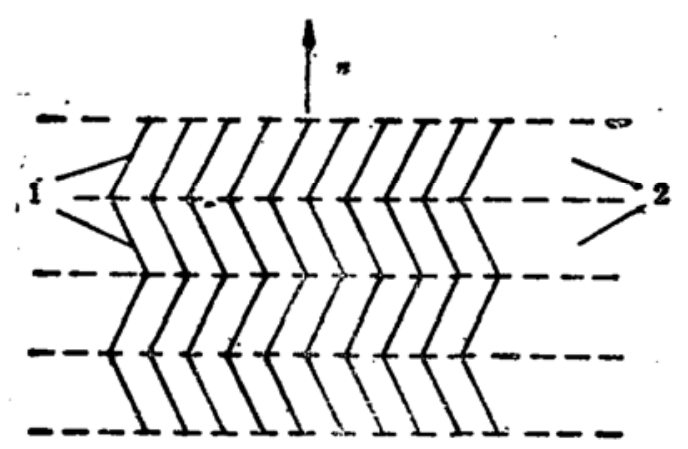

图 1 热致聚芳酯液晶态取向条带织构与其分子 链平均取向方向 $\boldsymbol{n}$ 之间关系示意图

1 为分子链, 2 为条带

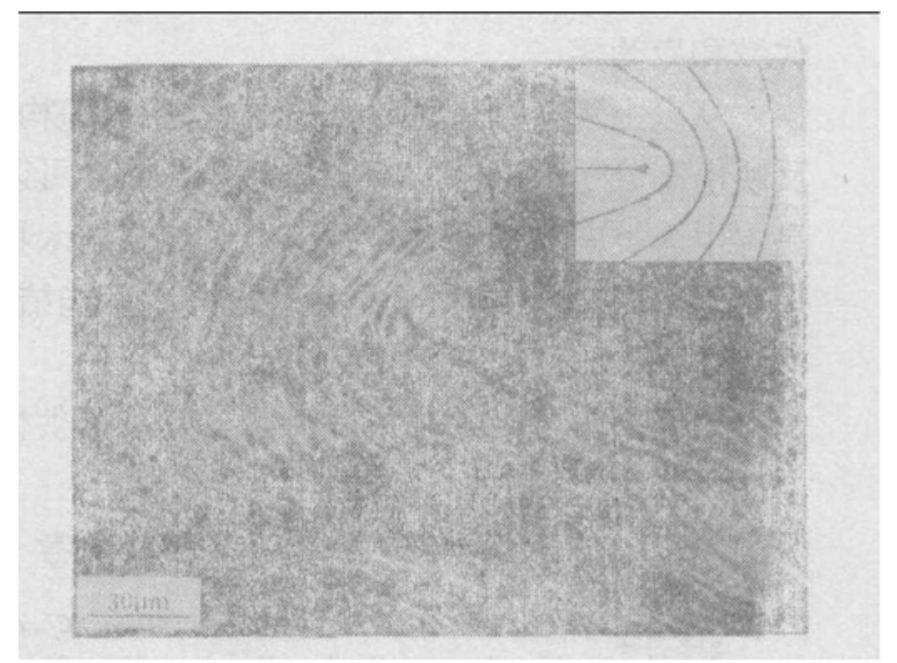

图 2 热致聚芳酯液晶态 $s=+\frac{1}{2}$ 向错结构的条 带织构装饰形态的偏光显微照相及落相对应的分子 指向矢取向排列图致

图 2 为聚芳酯液晶态经条带织构装饰后呈现郌度为 $s=+\frac{1}{2}$ 向错形态的 偏光显微照 相, 从图中可以清晰地看到围绕向错点有规则取向排列成 $s=+\frac{1}{2}$ 向错形态的条带 练构装 饰图. 根据图 1 中条带织构与分子链平均取向方向的关系, 可以画出艾相应的分子指向矢职 
向排列图象如图 2 中右上角所示. 图 3 为聚芳酯液晶态经条带织构装饰后呈现强度 $s=-\frac{1}{2}$ 向错形态的偏光显微照相, 从图中同样可以看到围绕向错点被条带织构装饰的 $s=-\frac{1}{2}$ 向 错结构的形态,其相对应的分子指向矢取向排列图象示于图 3 中右上角.

图 3 热致聚芳酯液晶态 $s=-\frac{1}{2}$ 向错结构的 条带织构装饰形态的偏光显微照相及其相对应的 分子指向矢取向排列图象

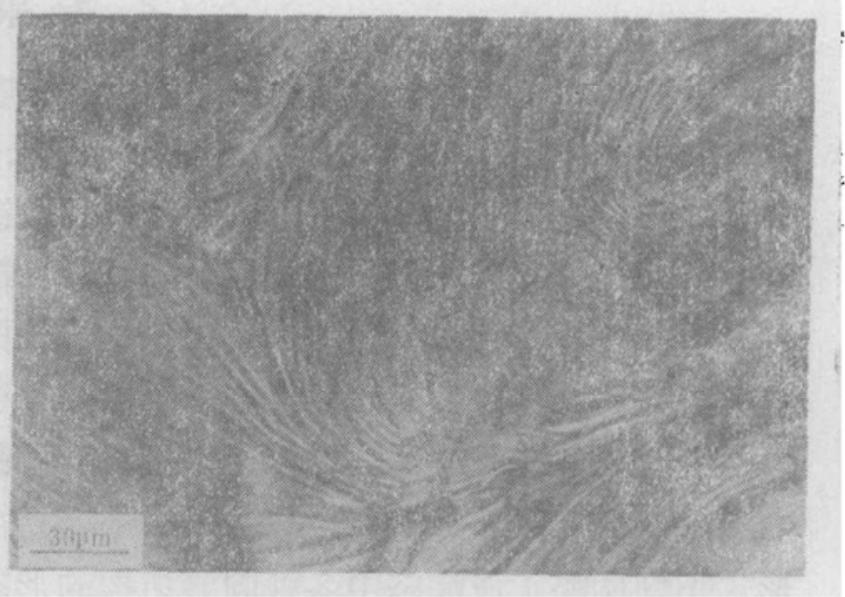

图 4 热致聚芳哯液晶态 $s= \pm \frac{1}{2}$ 问错对结构的条 带织构装饰形态的偏光显微照相

在高分子液晶态中所有的向错都不是单个孤立地存在，而必须与周围其他形式的向错相 互作用才能稳定存在,通常是同类相反符号的向错相互联接而形成的向错对如图 4 中所示.向 错之间相互作用的结果,有些向错消失或相互合并而形成新的向错,而在整个试样中向错的总 代数和应为零.

从以上观察结果可以得出结论,条带织构装饰技术与片晶装饰技术相似,同样可以清晰地 反映出高分子液晶态中的向错形态, 而且可以简便地应用普通偏光显微镜进行观察高分子液 晶态中各类向镜的形态. 条带织构装饰技术具有比片晶装饰技术制样简便和免受电子洙缩胒 损伤的优点, 尤其适用于结晶性不好或不能结晶的高分子液晶材料研究其缺陷结构.

致谢 清华大学化学系周其革教授提供样品,特表谢忱。

\section{參 考 文 献}

[ 1 ] Thomas, E. L., Wood, B. A., Faraday Disc. Chem. Soc., 1985, 79: 229.

[2] Wood, S. A., Thomas, E. L., Nature, 1986, 324: 655.

[ 3 ] Hudson, S. D., Thomas, E. L. et al., Mol. Cryst. Liq. Cryst., 1987, 153: 63,

[ 4] Food, J. R., Bassett, D. C. et al., Mol. Cryst. Liq. Eryst, 1990, 189B: 233.

[5] 蓝炎明、双红英, 高分子学报, 1991,(5): 584 .

[6] 陈寿䤦等,科学通报, 1992,37(14): 1284.

[7] Chen, S. X. et al., Mol. Cryst. Liq. Cryst., 1990, 128: 197.

[8] Chen, S. X. * al, Makromol. Chem, 1987, $188: 2317$. 